\title{
Computation of certain integral formulas involving generalized Wright function
}

\author{
Nabiullah Khan', Talha Usman², Mohd Aman', Shrideh Al-Omari ${ }^{3^{*}}$ (D) and Serkan Araci ${ }^{4}$ (I)
}

\section{"Correspondence:}

s.k.q.alomari@fet.edu.jo

${ }^{3}$ Department of Physics and Basic

Sciences, Faculty of Engineering

Technology, Al-Balqa Applied

University, Amman, 11134, Jordan

Full list of author information is

available at the end of the article

\begin{abstract}
The aim of the paper is to derive certain formulas involving integral transforms and a family of generalized Wright functions, expressed in terms of the generalized Wright hypergeometric function and in terms of the generalized hypergeometric function as well. Based on the main results, some integral formulas involving different special functions connected with the generalized Wright function are explicitly established as special cases for different values of the parameters. Moreover, a Gaussian quadrature formula has been used to compute the integrals and compare with the main results by using graphical representations.

Keywords: Generalized Wright function; Wright hypergeometric function; Mittag-Leffler function; Lavoie-Trottier integral formula; Generalized hypergeometric function; Gamma function
\end{abstract}

\section{Springer}

\section{Introduction and preliminaries}

The research on integral transforms involving special functions (see, e.g., [1-4, 6, 8$12,19])$ has received a considerable attention of the research community primarily because their application has made prominent contributions in several domains of mathematics, engineering and their applications in mathematical physics (see, e.g., [7, 15, 21, 22, 2730]). Among these special functions of mathematical physics, the Wright, the Bessel, and similar functions are of central importance and are fairly useful in the theory of integral and fractional calculus. To this end, a very little or no work on integral transforms involving Wright function has been done so far. In the main section, our focal point is to derive two essential theorems concerning integral transforms which will be used further, followed by related corollaries. In the last section, we specifically evaluate the Wright type auxiliary functions and some other deducible functions to derive a few results as special cases.

Now we recall some useful definitions of functions that are essential for the present investigation.

(c) The Author(s) 2020. This article is licensed under a Creative Commons Attribution 4.0 International License, which permits use, sharing, adaptation, distribution and reproduction in any medium or format, as long as you give appropriate credit to the original author(s) and the source, provide a link to the Creative Commons licence, and indicate if changes were made. The images or other third party material in this article are included in the article's Creative Commons licence, unless indicated otherwise in a credit line to the material. If material is not included in the article's Creative Commons licence and your intended use is not permitted by statutory regulation or exceeds the permitted use, you will need to obtain permission directly from the copyright holder. To view a copy of this licence, visit http://creativecommons.org/licenses/by/4.0/. 
Definition 1.1 Wright introduced the classical Wright function $W_{\alpha, \beta}(t)[13,20]$, defined by the series representation

$$
W_{\alpha, \beta}(t)=\sum_{n=0}^{\infty} \frac{1}{\Gamma(\alpha n+\beta)} \frac{t^{n}}{n !} \quad(\beta \in \mathbb{C}, \alpha>-1),
$$

$\forall \alpha \in \mathbb{R}$ and $\beta, \gamma, \delta \in \mathbb{C} ; \alpha>-1, \delta \neq 0,-1,-2, \ldots$, with $t \in \mathbb{C}$ and $|t|<1$ with $\alpha=-1$, a further generalization of the Wright function (see, for details, [18]) was introduced as

$$
W_{\alpha, \beta}^{\gamma, \delta}(t)=\sum_{n=0}^{\infty} \frac{(\gamma)_{n}}{(\delta)_{n} \Gamma(\alpha n+\beta)} \frac{t^{n}}{n !}
$$

where $(\gamma)_{n}$ is the Pochhammer symbol (see [21] p. 2 and pp. 4-6) and $\Gamma(\cdot)$ is the gamma function (see [21] Sect. 1.1), with the two auxiliary functions

$$
M_{\alpha}^{\gamma, \delta}(t)=W_{-\alpha, 1-\alpha}^{\gamma, \delta}(-t)=\sum_{n=0}^{\infty} \frac{(\gamma)_{n}}{\Gamma(1-\alpha-\alpha n)(\delta)_{n}} \frac{(-t)^{n}}{n !}
$$

and

$$
F_{\alpha}^{\gamma, \delta}(t)=W_{-\alpha, 0}^{\gamma, \delta}(-t)=\sum_{n=1}^{\infty} \frac{(\gamma)_{n}}{\Gamma(-\alpha n)(\delta)_{n}} \frac{(-t)^{n}}{n !} .
$$

Wright [24-26] studied the generalized Wright hypergeometric function defined as

$$
{ }_{p} \psi_{q}\left[\begin{array}{l}
\left(\alpha_{1} A_{1}\right), \ldots,\left(\alpha_{p}, A_{1}\right) ; t \\
\left(\beta_{1}, B_{1}\right), \ldots,\left(\beta_{q}, B_{q}\right) ;
\end{array}\right]=\sum_{m=0}^{\infty} \frac{\prod_{j=1}^{p} \Gamma\left(\alpha_{j}+A_{j} m\right)}{\prod_{j=1}^{q} \Gamma\left(\beta_{j}+B_{j} m\right)} \frac{t^{m}}{m !},
$$

where the coefficients $\left(A_{1}, \ldots, A_{p} ; B_{1}, \ldots B_{q}\right) \in \mathbb{R}^{+}$obey

$$
1-\sum_{j=1}^{p} A_{j}+\sum_{j=1}^{q} B_{j} \geq 0 .
$$

It is easily seen that (5) is the generalization of the famous generalized hypergeometric series ${ }_{p} F_{q}$ defined by

$$
{ }_{p} F_{q}\left[\begin{array}{c}
\alpha_{1}, \ldots, \alpha_{p} ; \\
\beta_{1}, \ldots, \beta_{q} ;
\end{array}\right]=\sum_{n=0}^{\infty} \frac{\left(\alpha_{1}\right)_{n} \cdots\left(\alpha_{p}\right)_{n}}{\left(\beta_{1}\right)_{n} \cdots\left(\beta_{q}\right)_{n}} \frac{t^{n}}{n !} .
$$

The Fox H-function [16], a generalization of the Fox-Wright function, is defined in terms of the Mellin-Barnes integral as

$$
H_{\alpha, \beta}^{\mu, \sigma}\left[x \mid \begin{array}{l}
\left(a_{1}, A_{1}\right), \ldots \ldots,\left(a_{\alpha}, A_{\alpha}\right) \\
\left(b_{1}, B_{1}\right), \ldots \ldots,\left(b_{\beta}, B_{\beta}\right)
\end{array}\right]=\frac{1}{2 \pi i} \int_{L} \Theta(s) x^{-s} d s,
$$

where $i=(-1)^{\frac{1}{2}}, x \neq 0$ and $x^{-s}=\exp [-s \ln |x|+i \arg x]$ and

$$
\Theta(s)=\frac{\left\{\prod_{j=1}^{\sigma} \Gamma\left(1-a_{j}-A_{j} s\right)\right\}\left\{\prod_{j=1}^{\mu} \Gamma\left(b_{j}+B_{j} s\right)\right\}}{\left\{\prod_{j=\mu+1}^{\beta} \Gamma\left(1-b_{j}-B_{j} s\right)\right\}\left\{\prod_{j=\sigma+1}^{\alpha} \Gamma\left(a_{j}+A_{j} s\right)\right\}} .
$$


The Meijer G-function [5] introduced by Meijer is defined as

$$
\begin{aligned}
G_{\alpha, \beta}^{\mu, \sigma} & \left(\left.x\right|_{c_{1}, c_{2}, \ldots, c_{\beta}} ^{a_{1}, a_{2}, \ldots, a_{\alpha}}\right) \\
= & H_{\alpha, \beta}^{\mu, \sigma}\left[\left.x\right|_{\left(b_{k}, 1\right)_{1}^{\beta}} ^{\left(a_{k}, 1\right)_{1}^{\alpha}}\right]=\sum_{k=1}^{\mu} \frac{\prod_{j=1}^{\mu} \Gamma\left(c_{j}-c_{k}\right) \prod_{j=1}^{\sigma} \Gamma\left(1+c_{k}-a_{j}\right) x^{c_{k}}}{\prod_{j=\mu+1}^{\beta} \Gamma\left(1+c_{k}-c_{j}\right) \prod_{j=\sigma+1}^{\alpha} \Gamma\left(a_{j}-c_{k}\right)} \\
& \quad \times{ }_{p} F_{q}\left(1+c_{k}-a_{1}, \ldots, 1+c_{k}-a_{\alpha} ; 1+c_{k}-c_{1}, \ldots, 1+c_{k}-c_{\beta} ;(-1)^{\alpha-\mu-\sigma} x\right),
\end{aligned}
$$

where $1 \leq \mu \leq \beta, 0 \leq \sigma \leq \alpha \leq \beta-1$.

The classical Mittag-Leffler function [17, 23] is defined as

$$
E_{\alpha, \beta}(t)=\sum_{k=0}^{\infty} \frac{t^{k}}{\Gamma(k \alpha+\beta)} \quad(\alpha>0, \beta \in \mathbb{C}) .
$$

We also note that $W_{\alpha, \beta}^{\gamma, \delta}$ immediately reduces to the above-mentioned functions as follows:

1. On replacing $\alpha$ by $-\alpha, t$ by $-t$, setting $\beta=0$ in (2) and with the help of (4), we get

$$
W_{-\alpha, 0}^{\gamma, \delta}(-t)=F_{\alpha}^{\gamma, \delta}(t)
$$

2. On replacing $\alpha$ by $-\alpha, t$ by $-t$, setting $\beta=1-\alpha$ in (2) and with the help of (3), we get

$$
W_{-\alpha, 1-\alpha}^{\gamma, \delta}(-t)=M_{\alpha}^{\gamma, \delta}(t)
$$

3. On replacing $t$ by $-t$ in (2) and with the help of (7), we can easily relate the generalized Wright function with the Fox $\mathrm{H}$-function as

$$
\frac{\Gamma(\gamma)}{\Gamma(\delta)} W_{\alpha, \beta}^{\gamma, \delta}(-t)=H_{13}^{1} 1\left[t \mid \begin{array}{c}
(1-\gamma, 1) \\
(0,1),(1-\beta, \alpha),(1-\delta, 1)
\end{array}\right]
$$

4. On replacing $t$ by $-t, \alpha=1$ in (2) and with the help of (8), we can easily relate the generalized Wright function with the Meijer G-function as

$$
\frac{\Gamma(\gamma)}{\Gamma(\delta)} W_{\alpha, \beta}^{\gamma, \delta}(-t)=G_{13}^{1} \frac{1}{t}\left[\begin{array}{c}
1-\gamma \\
0,1-\beta, 1-\delta
\end{array}\right]
$$

5. On setting $\alpha=0, \gamma=1$ in (2) and with the help of (9), we get

$$
\frac{\Gamma(\beta)}{\Gamma(\delta)} W_{0, \beta}^{1, \delta}(t)=E_{1, \delta}(t)
$$

Definition 1.2 The following result is also essential for the present investigation. For $(\Re(u)>0$ and $\Re(v)>0)$, the Lavoie-Trottier [14] integral formula is defined as

$$
\int_{0}^{1} x^{u-1}(1-x)^{2 v-1}\left(1-\frac{x}{3}\right)^{2 u-1}\left(1-\frac{x}{4}\right)^{v-1} d x=\left(\frac{2}{3}\right)^{2 u} \frac{\Gamma(u) \Gamma(v)}{\Gamma(u+v)}
$$




\section{Main results}

In this section, considering the integral in (15) and the generalized Wright function in (2), we establish two theorems as follows.

Theorem 1 With the existence of the conditions $\alpha \in \mathbb{R}$ and $\beta, \gamma, \delta, u, v \in \mathbb{C}, \Re(u+v)>0, \alpha>$ $-1, \delta \neq 0,-1,-2, \ldots$ with $t \in \mathbb{C}$ and $|t|<1$ with $\alpha=-1$ and $x>0$, the following formula holds:

$$
\begin{aligned}
& \int_{0}^{1} x^{u+\nu-1}(1-x)^{2 u-1}\left(1-\frac{x}{3}\right)^{2(u+v)-1}\left(1-\frac{x}{4}\right)^{u-1} W_{\alpha, \beta}^{\gamma, \delta}\left\{t\left(1-\frac{x}{4}\right)(1-x)^{2}\right\} d x \\
& =\left(\frac{2}{3}\right)^{2(u+v)} \frac{\Gamma(u+v) \Gamma(\delta)}{\Gamma(\gamma)}{ }_{2} \psi_{3}\left[\begin{array}{lll}
(u, 1), & (\gamma, 1) ; & \\
(\delta, 1), & (\beta, \alpha), & (2 u+v, 1) ;
\end{array}\right] .
\end{aligned}
$$

Proof Applying the definition (2) in the left-hand side of (16) and denoting it by $\mathcal{I}$, we write

$$
\mathcal{I}=\sum_{n=0}^{\infty} \frac{t^{n}(\gamma)_{n}}{n !(\delta)_{n} \Gamma(\alpha n+\beta)} \int_{0}^{1} x^{u+\nu-1}(1-x)^{2 u+2 n-1}\left(1-\frac{x}{3}\right)^{2 u+2 v-1}\left(1-\frac{x}{4}\right)^{u+n-1} d x
$$

Using (15) and interchanging the order of integration and summation, we obtain

$$
\mathcal{I}=\left(\frac{2}{3}\right)^{2(u+v)} \frac{\Gamma(u+v) \Gamma(\delta)}{\Gamma(\gamma)} \sum_{n=0}^{\infty} \frac{\Gamma(u+n) \Gamma(\gamma+n)}{\Gamma(\delta+n) \Gamma(\alpha n+\beta) \Gamma(2 u+v+n)} \frac{t^{n}}{n !}
$$

which on using (5), yields the required assertion (16) of Theorem 1.

Theorem 2 With the conditions already mentioned in Theorem 1, the following formula holds:

$$
\begin{aligned}
& \int_{0}^{1} x^{u-1}(1-x)^{2 u+2 v-1}\left(1-\frac{x}{3}\right)^{2 u-1}\left(1-\frac{x}{4}\right)^{u+v-1} W_{\alpha, \beta}^{\gamma, \delta}\left\{x t\left(1-\frac{x}{3}\right)^{2}\right\} d x \\
& =\left(\frac{2}{3}\right)^{2 u} \frac{\Gamma(u+v) \Gamma(\delta)}{\Gamma(\gamma)}{ }_{2} \psi_{3}\left[\begin{array}{c}
(u, 1),(\gamma, 1) ; \\
(\delta, 1),(\beta, \alpha),(2 u+v, 1) ;
\end{array}\right] .
\end{aligned}
$$

Proof The result in (19) can be derived with ease by the same procedure as followed in the establishment of (16).

Based on the previous theorems, at least two corollaries immediately follow which exploit the use of the hypergeometric function in (6).

Corollary 2.1 Let $u+v \in \mathbb{C} \backslash \mathbb{Z}_{o}^{-}$and with all conditions of Theorem 1 , the following integral formula holds true:

$$
\begin{gathered}
\int_{0}^{1}(1-x)^{2 u-1} x^{u+v-1}\left(1-\frac{x}{3}\right)^{2 u+2 v-1}\left(1-\frac{x}{4}\right)^{u-1} W_{\alpha, \beta}^{\gamma, \delta}\left\{t\left(1-\frac{x}{4}\right)(1-x)^{2}\right\} d x \\
=\left(\frac{2}{3}\right)^{2 u+2 v} \frac{\Gamma(u+v) \Gamma(u)}{\Gamma(\beta) \Gamma(2 u+v)}{ }_{2} F_{\alpha+2}\left[\begin{array}{c}
(\gamma),(u) \\
\left.(\delta),(2 u+v),\left(\frac{\beta}{\alpha}\right),\left(\frac{\beta+1}{\alpha}\right) \ldots,\left(\frac{\beta+\alpha-1}{\alpha}\right) \mid \frac{t}{\alpha^{\alpha}}\right] .
\end{array}\right.
\end{gathered}
$$


Corollary 2.2 Let $u+v \in \mathbb{C} \backslash \mathbb{Z}_{o}^{-}$and with all conditions of Theorem 1 , the following integral formula holds true:

$$
\begin{aligned}
& \int_{0}^{1}(1-x)^{2 u+2 v-1} x^{u-1}\left(1-\frac{x}{3}\right)^{2 u-1}\left(1-\frac{x}{4}\right)^{u+v-1} W_{\alpha, \beta}^{\gamma, \delta}\left\{t x\left(1-\frac{x}{3}\right)\right\} d x \\
& =\left(\frac{2}{3}\right)^{2 u+2 v} \frac{\Gamma(u+v) \Gamma(u)}{\Gamma(\beta) \Gamma(2 u+v)_{2}} F_{\alpha+2}\left[\begin{array}{c}
(\gamma),(u) \\
\left.(\delta),(2 u+v),\left(\frac{\beta}{\alpha}\right),\left(\frac{\beta+1}{\alpha}\right), \ldots,\left(\frac{\beta+\alpha-1}{\alpha}\right) \mid \frac{4 t}{9 \alpha^{\alpha}}\right] .
\end{array}\right.
\end{aligned}
$$

Proof Making use of the result (for $n \in \mathbb{N}_{o}$ )

$$
(\lambda)_{2 n}=\left(\frac{\lambda}{2}\right)_{n} 2^{2 n}\left(\frac{\lambda+1}{2}\right)_{n}
$$

in (18) and with the help of (5), we can easily establish (20). A similar approach will establish (21).

\section{Special cases}

According to the procedure we have employed, we can appreciate the importance of the special cases mentioned in (10)-(14) to establish some new results. We have indeed the following result.

Corollary 3.1 $\forall u, v, b \in \mathbb{C}$ with $\Re(u)>\frac{b}{2}, \Re(u+v)>0, \Re(2 u+v)>\frac{b}{2}, \alpha \in(0,1)$ and $x>0$, let

$$
\begin{gathered}
\int_{0}^{1}(1-x)^{2 u-b-1} x^{u+v-1}\left(1-\frac{x}{3}\right)^{2 u+2 v-1}\left(1-\frac{x}{4}\right)^{u-\frac{b}{2}-1} F_{\alpha}^{\gamma, \delta}\left\{t\left(1-\frac{x}{4}\right)(1-x)^{2}\right\} d x \\
=\left(\frac{2}{3}\right)^{2 u+2 v} \frac{\Gamma(u+v) \Gamma(\delta)}{\Gamma(\gamma)}{ }_{2} \psi_{3}\left[\begin{array}{c}
(\gamma, 1),\left(u-\frac{b}{2}, 1\right) ; \\
(\delta, 1),(0,-\alpha),\left(2 u+v-\frac{b}{2}, 1\right) ;
\end{array}\right] .
\end{gathered}
$$

Corollary 3.2 Allowing for the conditions already stated in (22), we have

$$
\begin{gathered}
\int_{0}^{1}(1-x)^{2 u+2 v-1} x^{u-\frac{b}{2}-1}\left(1-\frac{x}{3}\right)^{2 u-b-1}\left(1-\frac{x}{4}\right)^{u+v-1} F_{\alpha}^{\gamma, \delta}\left\{t x\left(1-\frac{x}{3}\right)^{2}\right\} d x \\
=\left(\frac{2}{3}\right)^{2 u-b} \frac{\Gamma(u+v) \Gamma(\delta)}{\Gamma(\gamma)}{ }_{2} \psi_{3}\left[\begin{array}{c}
(\gamma, 1),\left(u-\frac{b}{2}, 1\right) ; \\
(\delta, 1),(0,-\alpha),\left(2 u+v-\frac{b}{2}, 1\right) ;-\frac{4 t}{9}
\end{array}\right] .
\end{gathered}
$$

The proof of (22) and (23) is very similar to that of Theorem 1 and Theorem 2. It can be easily established by setting $\beta=0, \alpha=-\alpha$, and replacing $t$ by $-t$ and then using (10).

Corollary 3.3 Allowing for the conditions already stated in (22), we get

$$
\begin{gathered}
\int_{0}^{1}(1-x)^{2 u-b-1} x^{u+v-1}\left(1-\frac{x}{3}\right)^{2 u+2 v-1}\left(1-\frac{x}{4}\right)^{u-\frac{b}{2}-1} M_{\alpha}^{\gamma, \delta}\left\{t\left(1-\frac{x}{4}\right)(1-x)^{2}\right\} d x \\
=\left(\frac{2}{3}\right)^{2(u+v)} \frac{\Gamma(u+v) \Gamma(\delta)}{\Gamma(\gamma)}{ }_{2} \psi_{3}\left[\begin{array}{c}
(\gamma, 1),\left(u-\frac{b}{2}, 1\right) ; \\
(\delta, 1),(1-\alpha,-\alpha),\left(2 u+v-\frac{b}{2}, 1\right) ;
\end{array}\right] .
\end{gathered}
$$


Corollary 3.4 Allowing for the conditions already stated in (22), we get

$$
\begin{gathered}
\int_{0}^{1}(1-x)^{2 u+2 v-1} x^{u-\frac{b}{2}-1}\left(1-\frac{x}{3}\right)^{2 u-b-1}\left(1-\frac{x}{4}\right)^{u+v-1} M_{\alpha}^{\gamma, \delta}\left\{t x\left(1-\frac{x}{3}\right)^{2}\right\} d x \\
=\left(\frac{2}{3}\right)^{2 u-b} \frac{\Gamma(u+v) \Gamma(\delta)}{\Gamma(\gamma)}{ }_{2} \psi_{3}\left[\begin{array}{c}
(\gamma, 1),\left(u-\frac{b}{2}, 1\right) ; \\
(\delta, 1),(1-\alpha,-\alpha),\left(2 u+v-\frac{b}{2}, 1\right) ; 9
\end{array}\right] .
\end{gathered}
$$

Corollary 3.5 Allowing for the conditions already stated in (22), we get

$$
\begin{aligned}
& \int_{0}^{1}(1-x)^{2 u-b-1} x^{u+v-1}\left(1-\frac{x}{3}\right)^{2 u+2 v-1}\left(1-\frac{x}{4}\right)^{u-\frac{b}{2}-1} \\
& \quad \times H_{13}^{1} \frac{1}{3}\left[\left.\left(1-\frac{x}{4}\right)(1-x)^{2} t\right|_{(0,1),(1-\beta, \alpha),(1-\delta, 1)} ^{(1-\gamma, 1)}\right] d x \\
& =\left(\frac{2}{3}\right)^{2 u+2 v} \Gamma(u+v)_{2} \psi_{3}\left[\begin{array}{c}
(\gamma, 1),\left(u-\frac{b}{2}, 1\right) ; \\
(\delta, 1),(\beta, \alpha),\left(2 u+v-\frac{b}{2}, 1\right) ;
\end{array}\right] .
\end{aligned}
$$

Corollary 3.6 Allowing for the conditions already stated in (22), we get

$$
\begin{aligned}
& \int_{0}^{1} x^{u-\frac{b}{2}-1}(1-x)^{2(u+v)-1}\left(1-\frac{x}{3}\right)^{2 u-b-1}\left(1-\frac{x}{4}\right)^{u+v-1} \\
& \times H_{13}^{1} \frac{1}{3}\left[x t\left(1-\frac{x}{3}\right)^{2} \mid \begin{array}{c}
(1-\gamma, 1) \\
(0,1),(1-\beta, \alpha),(1-\delta, 1)
\end{array}\right] d x \\
& =\left(\frac{2}{3}\right)^{2 u-b} \Gamma(u+v)_{2} \psi_{3}\left[\begin{array}{c}
(\gamma, 1),\left(u-\frac{b}{2}, 1\right) ; \\
(\delta, 1),(\beta, \alpha),\left(2 u+v-\frac{b}{2}, 1\right) ;-\frac{4 t}{9}
\end{array}\right] .
\end{aligned}
$$

Corollary 3.7 Allowing for the conditions already stated in (22), we get

$$
\begin{aligned}
& \int_{0}^{1} x^{u+v-1}(1-x)^{2 u-b-1}\left(1-\frac{x}{3}\right)^{2(u+v)-1}\left(1-\frac{x}{4}\right)^{u-\frac{b}{2}-1} \\
& \quad \times G_{1}^{1} \frac{1}{3}\left[\left(1-\frac{x}{4}\right)(1-x)^{2} t \mid \begin{array}{c}
1-\gamma \\
0,1-\beta, 1-\delta
\end{array}\right] d x \\
& =\left(\frac{2}{3}\right)^{2(u+v)} \Gamma(u+v)_{2} \psi_{3}\left[\begin{array}{c}
(\gamma, 1),\left(u-\frac{b}{2}, 1\right) ; \\
(\delta, 1),(\beta, 1),\left(2 u+v-\frac{b}{2}, 1\right) ;
\end{array}\right] .
\end{aligned}
$$

Corollary 3.8 Allowing for the conditions already stated in (22), the following integral formula holds true:

$$
\begin{aligned}
& \int_{0}^{1}(1-x)^{2 u+2 v-1} x^{u-\frac{b}{2}-1}\left(1-\frac{x}{3}\right)^{2 u-b-1}\left(1-\frac{x}{4}\right)^{u+v-1} \\
& \quad \times G_{13}^{11}\left[x t\left(1-\frac{x}{3}\right)^{2} \mid \begin{array}{c}
(1-\gamma, 1) \\
(0,1),(1-\beta, \alpha),(1-\delta, 1)
\end{array}\right] d x \\
& =\left(\frac{2}{3}\right)^{2 u-b} \Gamma(u+v)_{2} \psi_{3}\left[\begin{array}{c}
(\gamma, 1),\left(u-\frac{b}{2}, 1\right) ; \\
\left.(\delta, 1),(\beta, 1),\left(2 u+v-\frac{b}{2}, 1\right) ;-\frac{4 t}{9}\right] .
\end{array}\right.
\end{aligned}
$$


Figure 1 Solution of (16) with the parameters used in the simulation $u=3, v=3, \delta=5, \alpha=-1, \beta=12$ and $\gamma=2$

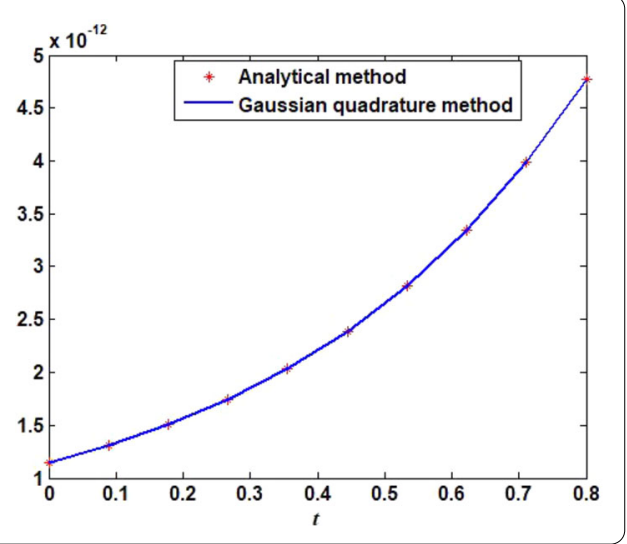

Figure 2 Solution of (19) with the parameters used in the simulation $u=6, v=3, \delta=5, \alpha=-1, \beta=12$ and $\gamma=2$

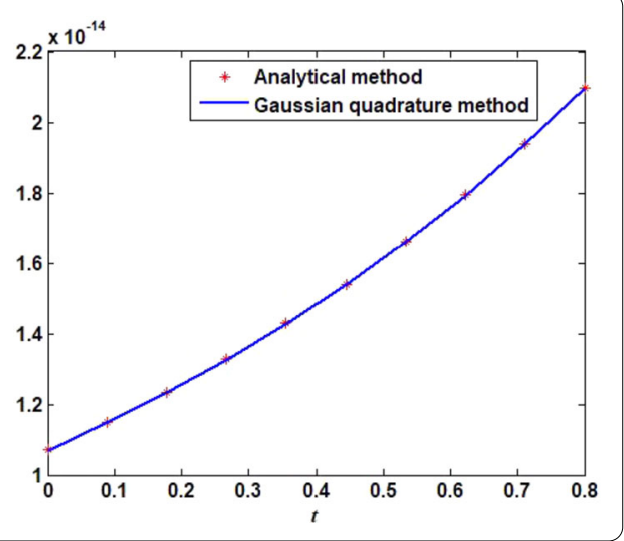

Corollary 3.9 Allowing for the conditions already stated in (22), we get

$$
\begin{aligned}
& \int_{0}^{1}(1-x)^{2 u-b-1} x^{u+v-1}\left(1-\frac{x}{3}\right)^{2 u+2 v-1}\left(1-\frac{x}{4}\right)^{u-\frac{b}{2}-1} E_{1, \delta}\left\{t\left(1-\frac{x}{4}\right)(1-x)^{2}\right\} d x \\
& =\left(\frac{2}{3}\right)^{2(u+v)} \Gamma(u+v)_{2} \psi_{2}\left[\begin{array}{c}
(1,1),\left(u-\frac{b}{2}, 1\right) ; \\
(\delta, 1),\left(2 u+v-\frac{b}{2}, 1\right) ;
\end{array}\right] .
\end{aligned}
$$

Corollary 3.10 Allowing for the conditions already stated in (22), we get

$$
\begin{aligned}
& \int_{0}^{1}(1-x)^{2 u+2 v-1} x^{u-\frac{b}{2}-1}\left(1-\frac{x}{3}\right)^{2 u-b-1}\left(1-\frac{x}{4}\right)^{u+v-1} E_{1, \delta}\left\{t x\left(1-\frac{x}{3}\right)^{2}\right\} d x \\
& =\left(\frac{2}{3}\right)^{2 u-b} \Gamma(u+v)_{2} \psi_{2}\left[\begin{array}{c}
(1,1),\left(u-\frac{b}{2}, 1\right) ; \\
(\delta, 1),\left(2 u+v-\frac{b}{2}, 1\right) ; \frac{4 t}{9}
\end{array}\right] .
\end{aligned}
$$

\section{Graphical interpretation}

In this section, we illustrate the solutions (16), (19), (22), (23), (24) and (25) using a graphical representation in terms of the parameter $t$. We use the numerical method named the Gaussian quadrature method to evaluate the integral of these equations and compare with the main results. To get the specific results, we take $n=8$ and $k=5$ (see Figs. 1-6). 
Figure 3 Solution of (22) with the parameters used in the simulation $u=6, v=3, \delta=5, \alpha=0.05, \beta=0$, $\gamma=5$ and $b=4$

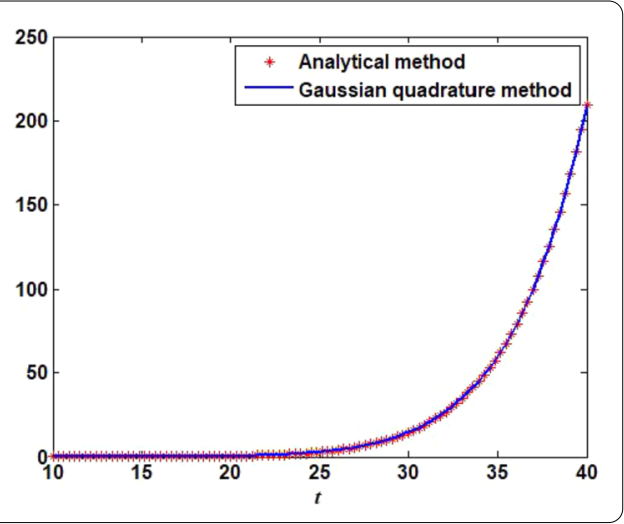

Figure 4 Solution of (23) with the parameters used in the simulation $u=6, v=3, \delta=5, \alpha=0.5, \beta=0, \gamma$ $=5$ and $b=-6$

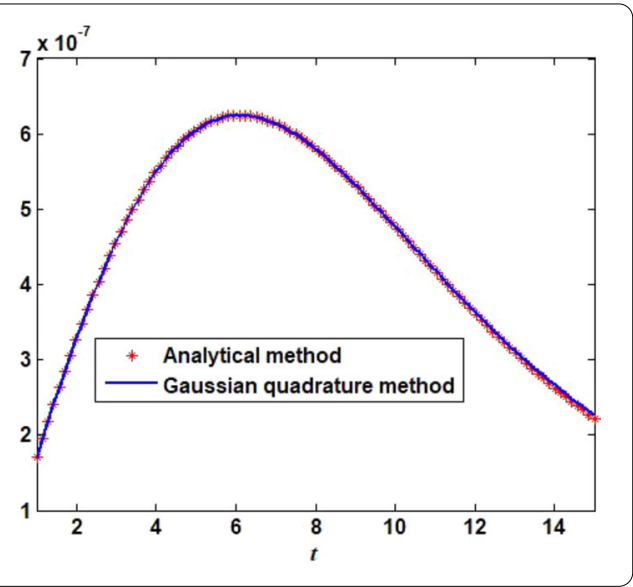

Figure 5 Solution of (24) with the parameters used in the simulation $u=6, v=3, \delta=10, \alpha=0.5, \gamma=12$ and $b=4$

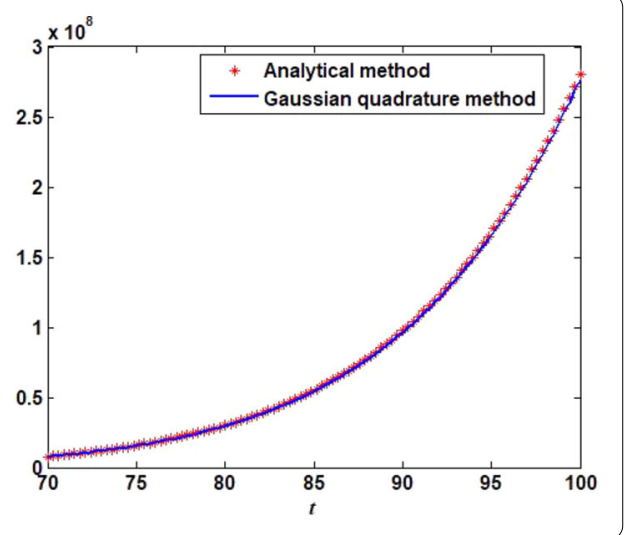

\section{Conclusion}

In these final remarks, it is worth stressing that the integral formulas computed in this paper involving a generalized Wright function are amenable for further research and generalizations. It is natural to note that the generalized Wright function depicts a close connection with several important special functions mentioned in the paper. As a consequence, we have attempted to compute the integrals of the above-mentioned functions in the form of generalized Wright function by some suitable parametric replacement, linking different families of special functions. We have also used the Gaussian quadrature formula to 
Figure 6 Solution of (24) with the parameters used in the simulation $u=6, v=3, \delta=10, \alpha=0.5, \gamma=12$ and $b=4$. Solution of (25) with the parameters used in the simulation $u=6, v=3, \delta=5, \alpha=0.5, \gamma=5$ and $b=-6$

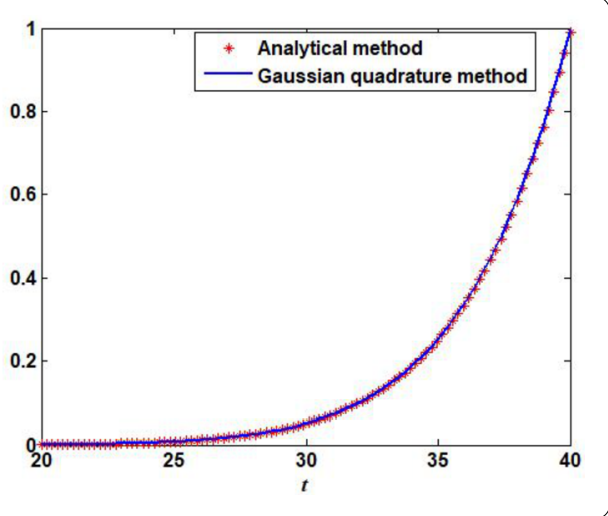

compare our main results graphically using Matlab. The conclusion we may draw is that the generalized Wright function has a wide range of applications in different domains; therefore, the results obtained provide a significant step which can yield some potential applications in the field of classical and applied mathematics.

\section{Acknowledgements}

This work was supported by the Science and Engineering Research Board (SERB), DST, Government of India (Gol), for the project under the Mathematical Research Impact Centric Support (MATRICES) with reference no. MTR/2017/000821.

Funding

No funding sources to be declared.

Availability of data and materials

Please contact the authors for data requests.

Competing interests

Authors declare they have no competing interests regarding the publication of the article.

\section{Authors' contributions}

The authors contributed equally in writing the present paper and performed all the steps of the proofs in this research. All authors read and approved the final manuscript.

\section{Author details}

'Department of Applied Mathematics, Faculty of Engineering and Technology, Aligarh Muslim University, Aligarh, 202002, India. ${ }^{2}$ Department of Mathematics, School of Basic and Applied Sciences, Lingaya's Vidyapeeth, Faridabad, 121002, Haryana, India. ${ }^{3}$ Department of Physics and Basic Sciences, Faculty of Engineering Technology, Al-Balqa Applied University, Amman, 11134, Jordan. ${ }^{4}$ Department of Economics, Faculty of Economics, Administrative and Social Sciences, Hasan Kalyoncu University, TR-27410, Gaziantep, Turkey.

\section{Publisher's Note}

Springer Nature remains neutral with regard to jurisdictional claims in published maps and institutional affiliations.

Received: 6 July 2020 Accepted: 6 September 2020 Published online: 15 September 2020

\section{References}

1. Al-Omari, S.K.Q.: Spaces for a class of Whittaker integral transformations. Kuwait J. Sci. 43(4), 32-38 (2016)

2. Al-Omari, S.K.Q.: Estimation of a modified integral associated with a special function kernel of Fox's H-function type. Commun. Korean Math. Soc. 35(1), 125-136 (2020)

3. Al-Omari, S.K.Q., Baleanu, D.: On the generalized Stieltjes transform of Fox's kernel function and its properties in the space of generalized functions. J. Comput. Anal. Appl. 23(1), 108-118 (2017)

4. Al-Omari, S.Q., Jumah, G., Al-Omari, J., Saxena, D.: A new version of the generalized Krätzel-Fox integral operators Mathematics 6, 222 (2018). https://doi.org/10.3390/math6110222

5. Andrews, L.C.: Special Functions for Engineers and Applied Mathematicians. MacMillan, New York (1985)

6. Ansari, A.: On the Fourier transform of the products of M-Wright functions. Bol. Soc. Parana. Mat. 33(1), 247-256 (2015)

7. Bira, B., Sekhar, T.R., Zeidan, D.: Exact solutions for some time-fractional evolution equations using Lie group theory. Math. Methods Appl. Sci. 41(16), 6717-6725 (2018)

8. Choi, J., Agarwal, P.: Certain unified integrals involving a product of Bessel functions of first kind. Honam Math. J. 35 667-677 (2013) 
9. Khan, N.U., Ghayasuddin, M., Usman, T.: On certain integral formulas involving the product of Bessel function and Jacobi polynomial. Tamkang J. Math. 47(3), 339-349 (2016)

10. Khan, N.U., Usman, T., Ghayasuddin, M.: A unified double integral associated with Whittaker functions. J. Nonlinear Syst. Appl. 5, 21-24 (2016)

11. Khan, N.U., Usman, T., Ghayasuddin, M.: Some integrals associated with multiindex Mittag-Leffler functions. J. Appl. Math. Inform. 34(3), 249-255 (2016)

12. Khan, N.U., Usman, T., Ghayasuddin, M.: A note on integral transforms associated with Humbert's confluent hypergeometric function. Electron. J. Math. Anal. Appl. 4(2), 259-265 (2016)

13. Kiryakova, V:: Some special functions related to fractional calculus and fractional (non-integer) order control systems and equations. Facta Univ. Ser. Autom. Control Robot. 7(1), 79-98 (2008)

14. Lavoie, J.L., Trottier, G.: On the sum of certain Appell's series. Ganita 20, 31-32 (1969)

15. Mandal, H., Bira, B., Zeidan, D.: Power series solution of time-fractional Majda-Biello system using Lie group analysis. In: Proceedings of International Conference on Fractional Differentiation and Its Applications (ICFDA) (2018)

16. Mathai, A.M., Saxena, R.K., Haubold, J.J.: The H-Function: Theory and Applications. Springer, Dordrecht (2010)

17. Mittag-Leffler, G.M.: Sur la nouvellefonction $E_{\alpha}(x)$. C. R. Math. Acad. Sci. 137, 554-558 (1903)

18. Moustafa, E.-S., Salem, A.: An extension of Wright function and its properties, Hindavi publishing corporation. J. Math. 2015, Article ID 950728 (2015)

19. Nisar, K.S., Suthar, D.L., Purohit, S.D., Aldhaifallah, M.: Some unified integral associated with the generalized Struve function. Proc. Jangjeon Math. Soc. 20(2), 261-267 (2017)

20. Podlubny, l.: Fractional Differential Equations. Academic Press, New York (1999)

21. Srivastava, H.M., Choi, J.: Zeta and q-Zeta Functions and Associated Series and Integrals. Elsevier, Amsterdam (2012)

22. Sultana, F., Singh, D., Pandey, R.K., Zeidan, D.: Numerical schemes for a class of tempered fractional integro-differential equations. Appl. Numer. Math. 157, 110-134 (2020)

23. Wiman, A.: Über den Fundamentalsatz in der Theorie der Funktionen $E_{a}(x)$. Acta Math. 29, 191-201 (1905)

24. Wright, E.M.: The asymptotic expansion of the generalized hypergeometric functions. J. Lond. Math. Soc. 10, 286-293 (1935)

25. Wright, E.M.: The asymptotic expansion of integral functions defined by Taylor series. Philos. Trans. R. Soc. Lond. A 238, 423-451 (1940)

26. Wright, E.M.: The asymptotic expansion of generalized hypergeometric function II. Proc. Lond. Math. Soc. (2) 46, 389-408 (1940)

27. Zeidan, D., Bähr, P., Farber, P., Gräbel, J., Ueberholz, P.: Numerical investigation of a mixture two-phase flow model in two-dimensional space. Comput. Fluids 181, 90-106 (2019)

28. Zeidan, D., Bira, B.: Weak shock waves and its interaction with characteristic shocks in polyatomic gas. Math. Methods Appl. Sci. 42(14), 4679-4687 (2019)

29. Zeidan, D., Chau, C.K., Lu, T-T., Zheng, W-Q.: Mathematical studies of the solution of Burgers' equations by a domian decomposition method. Math. Methods Appl. Sci. 43(5), 2171-2188 (2020)

30. Zeidan, D., Chau, C.K., Lu, T.-T.: On the characteristic A domian decomposition method for the Riemann problem. Math. Methods Appl. Sci. (2019)

\section{Submit your manuscript to a SpringerOpen ${ }^{\circ}$ journal and benefit from:}

- Convenient online submission

- Rigorous peer review

Open access: articles freely available online

- High visibility within the field

Retaining the copyright to your article

Submit your next manuscript at $\gg$ springeropen.com 